\title{
A SEARCH FOR LYMAN BREAK GALAXIES AT $z>8$ IN THE NICMOS PARALLEL IMAGING SURVEY ${ }^{1}$
}

\author{
Alaina L. Henry, ${ }^{2}$ Matthew A. Malkan, ${ }^{2}$ James W. Colbert, ${ }^{3}$ Brian Siana, ${ }^{3}$ Harry I. Teplitz, ${ }^{3}$ \\ PATRICK MCCARThY, ${ }^{4}$ AND LIN YAN ${ }^{3}$ \\ Received 2006 October 19; accepted 2006 December 26; published 2007 January 22
}

\begin{abstract}
We have selected $14 J$-dropout Lyman break galaxy (LBG) candidates with $J_{110}-H_{160} \geq 2.5$ from the NICMOS Parallel Imaging Survey. This survey consists of $135 \mathrm{arcmin}^{2}$ of imaging in 228 independent sight lines, reaching average $5 \sigma$ sensitivities of $J_{110}=25.8$ and $H_{160}=25.6(\mathrm{AB})$. Distinguishing these candidates from dust-reddened star-forming galaxies at $z \sim 2-3$ is difficult and will require longer wavelength observations. We consider the likelihood that any $J$-dropout LBGs exist in this survey and find that if $L_{z=9.5}^{*}$ is significantly brighter than $L_{z=6}^{*}$ (a factor of 4), then a few $J$-dropout LBGs are likely. A similar increase in luminosity has been suggested by Eyles et al. and Yan et al., but the magnitude of this increase is uncertain.
\end{abstract}

Subject headings: galaxies: evolution — galaxies: formation — galaxies: high-redshift

\section{INTRODUCTION}

Searches for Lyman break galaxies (LBGs) have measured their luminosity functions (LFs) out to high redshifts $(z=$ 4-5; Kashikawa et al. 2006; Yoshida et al. 2006). This indicates that substantial numbers of galaxies were in place at $z=6$ and that galaxy formation must be ongoing at higher redshifts. Yan et al. (2006) and Eyles et al. (2007) have shown that the existing mass in $i$-dropouts at $z=6$ cannot be assembled unless star formation rates were higher in the past. The implied rapid first bursts of star formation at $z>7$ could produce galaxies with large enough rest-wavelength UV luminosities to be detected in the near-infrared. In addition, it is currently uncertain if the amount of star formation at $z=6$ is adequate to reionize the universe. $J$-dropout LBGs may play an important role in this process, which is likely between $9 \leq z \leq 14$ (Spergel et al. 2006).

The NICMOS Parallel Imaging Survey (Henry et al. 2005; A. L. Henry et al. 2007, in preparation) provides the unique combination of sensitivity and wide-area coverage required to find rare, luminous LBGs with $8 \leq z \leq 11$. With $135 \operatorname{arcmin}^{2}$ in 228 independent sight lines, we cover an order of magnitude more sky than the NICMOS portions of Hubble Deep FieldNorth (HDF-N; Dickinson 1999; Thompson et al. 1999) and the Ultra Deep Field (Thompson et al. 2005). Although the NICMOS Parallel Imaging Survey is less sensitive than these surveys, the galaxies presented here are better suited for followup observations. In addition, it surpasses most ground-based imaging.

We have identified a sample of possible $J$-dropout galaxies from the NICMOS Parallel Imaging Survey, and here we discuss the likelihood that any of them are at $z \geq 8$ as well as the possible implications for star formation near the epoch of reionization. We use $H_{0}=71 \mathrm{~km} \mathrm{~s}^{-1} \mathrm{Mpc}^{-1}, \Omega_{\Lambda}=0.73$, and $\Omega_{M}=0.27$,

\footnotetext{
${ }^{1}$ Based on observations made with the NASA/ESA Hubble Space Telescope, obtained from the Space Telescope Science Institute, which is operated by the Association of Universities for Research in Astronomy, Inc., under NASA contract NAS 5-26555. These observations are associated with proposals 9484, 9865, and 10226.

${ }^{2}$ Department of Physics and Astronomy, University of California, Los Angeles, CA; ahenry@astro.ucla.edu, malkan@astro.ucla.edu.

${ }^{3}$ Spitzer Science Center, California Institute of Technology, Pasadena, CA; colbert@ipac.caltech.edu,bsiana@ipac.caltech.edu,hit@ipac.caltech.edu,lyan@ ipac.caltech.edu.

${ }^{4}$ Observatories of the Carnegie Institute of Washington, Pasadena, CA; pmc2@ociw.edu.
}

and $\mathrm{AB}$ magnitudes are used throughout. We take $M_{1500}^{*}(z=$ $3)=-21.1$ (Steidel et al. 1999), which corresponds to $H_{160}=26.4$ at $z=9.5$.

\section{OBSERVATIONS AND SOURCE SELECTION}

\subsection{The NICMOS Parallel Imaging Survey}

We conducted the NICMOS Parallel Survey in Hubble Space Telescope (HST) observing cycles 7, 11, 12, and 13. Images and slitless grism spectroscopy were obtained in the parallel mode, using the NICMOS3 camera (Thompson et al. 1998). Early results from the cycle 7 survey are presented in McCarthy et al. (1999) Yan et al. (1998, 1999, 2000), and Teplitz et al. (1998).

The cycles 11, 12, and 13 imaging data will be described in more detail in A. L. Henry et al. (2007, in preparation); we provide a summary here. Each field was observed with both the $J_{110}$ (F110W) and $H_{160}(\mathrm{~F} 160 \mathrm{~W})$ filters, with average integration times of $\sim 1500$ and $\sim 2000 \mathrm{~s}$. Images are drizzled according to Fruchter \& Hook (1997), and the resulting point-spread function has a FWHM 0.3". Photometry was performed with SExtractor (Bertin $\&$ Arnouts 1996) in dual image mode. The $H_{160}$ images were used to detect sources, and fluxes were measured from identical Kron (1980) apertures in both bands. We require 9 pixels above $1.5 \sigma$ for detection. We further require a $5 \sigma$ detection in $H_{160}$, as measured in a $0.8^{\prime \prime}$ diameter aperture. The average $5 \sigma$ sensitivities reached are $J_{110}=25.8$ and $H_{160}=25.6$. We detect 7700 objects; the color-magnitude diagram is shown in Figure 1 (left).

\subsection{J-Dropout Candidates}

In Figure 1 (right), we show Bruzual \& Charlot (2003) evolutionary tracks to which we have added an intergalactic medium foreground absorption component that is completely opaque beyond $z=6$, where $\operatorname{Ly} \alpha$ passes into the $J_{110}$ filter. In addition, we show examples of two local ultraluminous infrared galaxies (ULIRGs), Arp 220 and IRAS 03521+0028. For Arp 220, we compile the UV to near-infrared spectral energy distribution (SED) from Goldader et al. (2002), Surace et al. (2000), and the RC3 catalog. For IRAS 03521+0028, which has a very red rest-frame optical SED, only the $B I H K^{\prime}$ photometry from Surace et al. (2000) is available, so we do not extend this track beyond $z=2$.

To minimize contamination from lower redshift interlopers, we adopt the color selection $J_{110}-H_{160}>2.5$ for $J$-dropout can- 

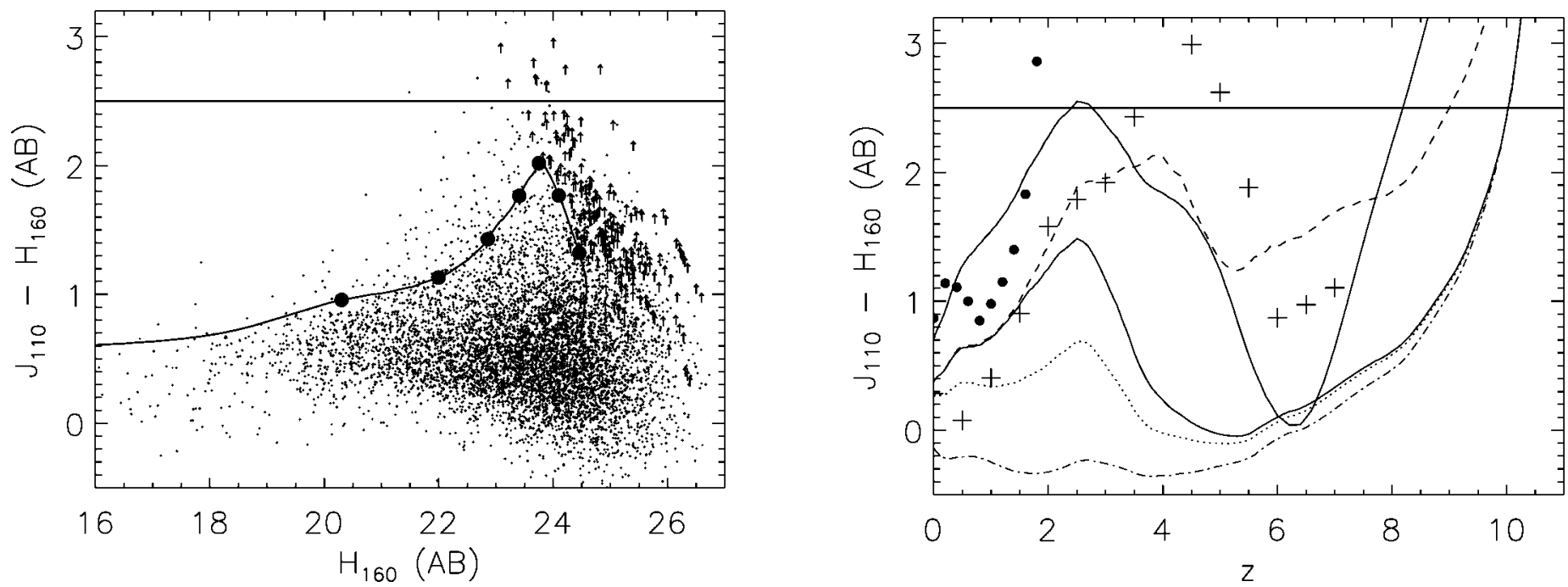

FIG. 1.-Left: NICMOS Parallel Imaging Survey color-magnitude diagram. All points are $5 \sigma$ detections, or better, in $H_{160}$. Arrows represent sources with no $J_{110}$ detection above $3 \sigma$. We overplot the Bruzual \& Charlot (2003) evolutionary track of a galaxy that formed at $z_{f}=12$, with a $A_{V}=1$, a Salpeter initial mass function, solar metallicity, and an exponentially declining star formation rate with a $500 \mathrm{Myr} e$-folding time. This model galaxy has a stellar mass of $7 \times 10^{10} M_{\odot}$ at $z=0$. The track is marked with fiducial redshift points at $z=0.5,1.0,1.5,2.0,2.5$ (peak), 3.0, and 3.5. Right: Curves show Bruzual \& Charlot (2003) evolutionary tracks. All models have a Salpeter initial mass function and solar metallicity. The bluest, dot-dashed track is a $10 \mathrm{Myr}$ old model with constant star formation and no extinction, while the remaining tracks are evolving, with $z_{f}=12$. Their star formation histories and extinctions are (dotted line) constant with $10 M_{\odot} \mathrm{yr}^{-1}$, and $A_{V}=0 ;$ (solid lines) exponentially declining, with a $0.5 \mathrm{Gyr} e$-folding time, and $A_{V}=0$ and 2; and (dashed line) an instantaneous burst with $A_{V}=0$. Extinction is added following the prescription by Cardelli et al. (1989), which contains a $2000 \AA$ bump. At $z \sim 6$, this feature is in the $H_{160}$ filter, the resulting dust obscured color is bluer than the intrinsic color. We also show the expected colors from two local ULIRG SEDs: Arp 220 (plus signs) and IRAS 03521+0028 (filled circles). The horizontal line indicates the color cut that we made at $J_{110}-H_{160} \geq 2.5$.

didates. Figure 1 (right) shows that the reddest $J$-dropout LBGs will be selected at $z \gtrsim 8$, while nearly all models meet this criterion at $z>10$. We find $14 J$-dropout candidates (Table 1); five are detected in $J_{110}$ (at $3 \sigma$ or better), but this does not disqualify them as LBGs, since some flux will fall in the $J_{110}$ filter for $z<10.5$. The remaining nine galaxies are among the sources that are plotted as lower limits (upward pointing arrows) in Figure 1. Possible contamination from lower redshift sources is discussed in $\S 3.1$.

Since exposure times varied from field to field, the NICMOS Parallel Imaging Survey is inhomogeneous in sensitivity. For selection of red galaxies, the faint limit of the sample is determined by the sensitivity in the $J_{110}$ images. For example, at $H_{160}=23.3,98 \%$ of the $J_{110}$ images have sufficient sensitivity for sources to be selected with $J_{110}-H_{160} \geq 2.5$. A halfmagnitude fainter, at $H_{160}=23.8$, this completeness drops to $\sim 50 \%$. Since fainter galaxies are more likely to be genuine $J$ dropout LBGs, we consider all galaxies with $J_{110}-H_{160} \geq$ 2.5 and correct for this incompleteness.

\section{DISCUSSION}

\subsection{Foreground Contamination}

Figure 1 (right) shows that some lower redshift galaxies may be selected as $J$-dropout candidates. Bouwens et al. (2005) find three galaxies with $J_{110}-H_{160} \geq 2.5$ in a $15 \operatorname{arcmin}^{2}$ survey. This density is consistent with the 14 sources we have found, since the NICMOS Parallel Imaging Survey covers 10 times the area of Bouwens et al., although with less sensitivity. Bouwens et al. use optical and $K$-band photometry to argue that these galaxies are all at lower redshift. Since they find no $J$ -

TABLE 1

$J$-Dropout LBG Candidates with $J_{110}-H_{160}>2.5$

\begin{tabular}{|c|c|c|c|c|c|c|}
\hline Identification & $H_{160}$ & $H_{160} \mathrm{~S} / \mathrm{N}$ & $J_{110}-H_{160}(\mathrm{AB})$ & R.A. (J2000.0) & Decl. (J2000.0) & $\begin{array}{l}\text { FWHM } \\
(\operatorname{arcsec})\end{array}$ \\
\hline ............ & 24.8 & 36 & $>2.7$ & 144616.09 & 403010.9 & 0.32 \\
\hline$\ldots \ldots \ldots$ & 23.2 & 26 & $>2.6$ & 221511.95 & -140801.0 & 0.55 \\
\hline $3 \ldots \ldots \ldots \ldots$ & 23.9 & 26 & $>2.6$ & $\begin{array}{lll}02 & 05 & 29.41\end{array}$ & -120555.1 & 0.39 \\
\hline $4 \ldots \ldots \ldots \ldots$ & 24.2 & 35 & $>2.7$ & $0231 \quad 17.39$ & $-0849 \quad 17.5$ & 0.30 \\
\hline $5 \ldots \ldots \ldots \ldots$ & 23.3 & 50 & 3.2 & 005936.34 & -511355.5 & 0.44 \\
\hline $6 \ldots \ldots \ldots \ldots$ & 23.9 & 30 & $>2.6$ & 074421.68 & 392141.1 & 0.42 \\
\hline $7 \ldots \ldots \ldots \ldots$ & 23.7 & 37 & $>2.8$ & 094839.80 & 673023.7 & 0.32 \\
\hline $8 \ldots \ldots \ldots \ldots$ & 24.0 & 43 & $>2.9$ & 104744.77 & 134658.1 & 0.46 \\
\hline$\ldots \ldots \ldots \ldots$ & 23.9 & 28 & 2.6 & $1534 \quad 13.04$ & 264824.3 & 0.36 \\
\hline ......... & 23.2 & 48 & 3.1 & 123542.37 & $62 \quad 1030.4$ & 0.38 \\
\hline ......... & 22.7 & 51 & 2.7 & 020957.31 & -505529.2 & 1.47 \\
\hline $12 \ldots \ldots \ldots$ & 23.7 & 27 & $>2.6$ & 210357.60 & 031628.2 & 0.37 \\
\hline $13 \ldots \ldots \ldots$ & 23.8 & 30 & 2.6 & $1758 \quad 07.12$ & 664519.9 & 0.49 \\
\hline $14 \ldots \ldots \ldots \ldots$ & 23.1 & 33 & $>2.8$ & 002542.99 & -122529.2 & 0.39 \\
\hline
\end{tabular}

Note. - Units of right ascension are hours, minutes, and seconds, and units of declination are degrees, arcminutes, and arcseconds. 


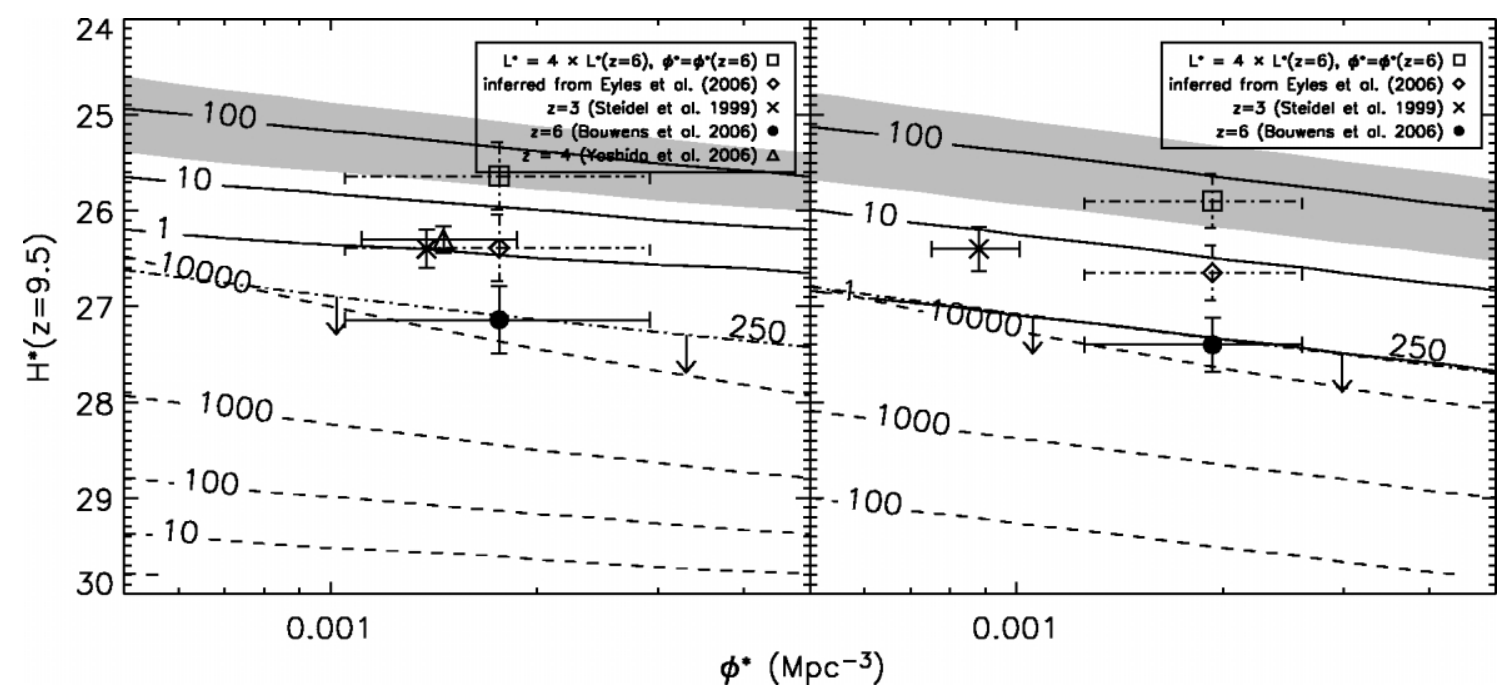

FIG. 2.-Contours of galaxies per square degree for this survey (solid lines) and a survey that is complete to 28.0 (dashed lines), calculated using a Schechter luminosity function (left) and a broken power law with the high- $L$ slope fixed at -4 (right). The gray region indicates $1-10$ objects in this 135 arcmin ${ }^{2}$ survey. The dot-dashed curve indicates $\$ 250 \mathrm{deg}^{-2}$ in the Bouwens et al. (2005) search for $J$-dropout LBGs. In the right panel, the dot-dashed curve overlaps the solid contour for $1 \mathrm{deg}^{-2}$. The filled circles indicate estimates of the LF at $z=9.5$, from lower redshift populations.

dropout LBGs with $J_{110}-H_{160} \geq 2.5$, the implied density is less than $250 \mathrm{deg}^{-2}$.

In addition, we consider the SEDs of two local ULIRGs. Daddi et al. (2005) have shown that ULIRGs have a surface density of about $1 \operatorname{arcmin}^{-2}$ at $z=2-3$. For Arp 220 $\left(L_{\text {bol }}=1.5 \times 10^{12} L_{\odot}\right)$, we expect $H_{160}=23.6$ at $z=2$, which is typical of the galaxies in this survey. However, ULIRGs like Arp 220 are not red enough at $z=2-3$ to be selected as part of this sample. In Figure 1 (right), we show the predicted color of another ULIRG, IRAS 03521+0028, which has a very red rest-frame optical SED. This galaxy is red enough to be selected at $z=2$, but it is doubtful if many ULIRGs at $z=2-3$ can be described by this red optical SED. If $\sim 10 \%$ display similar colors, this could account for the 14 $J$-dropout candidates. While galaxies like Arp 220 could also be selected by our color cut at $z \sim 4-5$, ULIRGs at these redshifts are expected to be rare, and would have to be very luminous, with $L_{\text {bol }} \geqslant \times 10^{13} L_{\odot}$. Even at $z=2-3$, the density of ULIRGs of this luminosity is low, with only $\sim 15 \mathrm{deg}^{-2}$ (Yan et al. 2005).

Contamination from Galactic sources is improbable. Dickinson et al. (2000) report on a similar point source in the HDF$\mathrm{N}$, with $J_{110}-H_{160}>2.3$, and find that it is unlikely a stellar or substellar source.

\subsection{Estimating the Density of Galaxies at $z \geq 8$}

Since no objects have ever been confirmed at less than $500 \mathrm{Myr}$ after the big bang, it is difficult to predict how bright or numerous they will be. Since small uncertainties in $L_{z=9.5}^{*}$ can have a large effect, we consider both a Schechter LF and a broken power law of the form

$$
\phi(m)=\frac{\phi^{*}}{10^{0.4\left(\alpha_{1}+1\right)\left(m-m^{*}\right)}+10^{0.4\left(\alpha_{2}+1\right)\left(m-m^{*}\right)}},
$$

with faint-end slope $\alpha_{1}$ and luminous slope $\alpha_{2} \cdot{ }^{5}$ In Figure 2,

\footnotetext{
${ }^{5}$ We fit the above broken power law to the $z=3$ population from Steidel et al. (1999) and the $z=6$ LBGs from Bouwens et al. (2006). We find $\alpha_{2} \sim-4$, and $\alpha_{1} \sim-1.6$ in both populations, so we hold both slopes constant.
}

we show contours in surface density, calculated by integrating the LF for a range of plausible $\phi^{*}$ and $H^{*}(z=9.5)$ :

$$
N=\int_{z} d z \frac{d V}{d z} \int_{m} P(m, z) \phi\left[m, m^{*}(z)\right] d m .
$$

This allows for the dimming of $H_{160}^{*}$ with redshift, which is large for $z>10.5$, where the $\operatorname{Ly} \alpha$ absorption passes into the $H_{160}$ filter. The quantity $d z(d V / d z)$ is the comoving volume element, and $P(m, z)$ corrects for galaxies that are missed because of incompleteness or because they are not red enough to be selected by our color cut. To estimate $P(m, z)$, we separate the quantity into $P(m, z)=p_{1}(m) p_{2}(z)$, taking $p_{1}(m)$ as the completeness, which we discussed in $\S 2.2$. For $p_{2}(z)$, we assume that galaxies are equally distributed between the reddest and bluest models in Figure 1 (right), ${ }^{6}$ so that $p_{2}(z)$ increases from zero to one between $8<z<10$.

Under these assumptions, the galaxies in this survey should lie between $8 \leq z \leq 11$, with an average redshift of $z=9-10$, depending on the chosen LF. We therefore adopt an average redshift of $z=9.5$. We repeat this calculation to estimate a constraint on the LF based on the upper limit in surface density found in the Bouwens et al. (2005) search for $J$-dropouts, which consists of six fields imaged to an average sensitivity of $H_{160}=28.0$. Like the NICMOS Parallel Imaging Survey, the Bouwens et al. (2005) survey is limited by sensitivity in $J_{110}$, so that galaxies with $J_{110}-H_{160} \geq 2.5$ are only selected down to $H_{160} \sim 25.5$. Their finding of less than $250 \mathrm{deg}^{-2}$ is plotted as the dot-dashed line in Figure 2. Also, for comparison to more sensitive surveys, we show surface densities calculated with $p_{1}(m)=1$ and a detection limit of $H_{160}=28$ (this requires a sensitivity of $J_{110}=30.5$, which can be reached in less than $10 \mathrm{hr}$ of integration with the James Webb Space Telescope).

These plots show the expected density of $J$-dropout LBGs, based on a given $H^{*}$ and $\phi^{*}$. The shaded area of Figure 2 shows

\footnotetext{
${ }^{6}$ This is consistent with the results of Thompson (2003) and Thompson et al. (2006), which find that high-redshift galaxies are best fit by the bluest spectral types. Even the reddest model used here is only a few hundred megayears old at $z=8$ and is therefore comparable to the blue models used by Thompson et al.
} 
the allowed region for $H^{*}$ and $\phi^{*}$ if one to $10 \mathrm{~J}$-dropout LBGs exist in this survey. If none of the candidates are $z \geq 8$ LBGs, then $H^{*}$ and $\phi^{*}$ should be below the shaded region. We show examples from a few observed LFs, including the broken powerlaw fits that we made. If the $z=6$ measurement by Bouwens et al. (2006) is a good indicator of the population at $z \sim 9.5$, then the density of sources that we expect is of order $0.01 \mathrm{deg}^{-2}$ in the Schechter form, and about $1 \mathrm{deg}^{-2}$ in the broken powerlaw LF. These densities are too low to suggest any $J$-dropout LBGs in the NICMOS Parallel Imaging Survey, which covers only $0.04 \mathrm{deg}^{2}$. On the other hand, Richard et al. (2006) in a lensing survey for $J$-dropout galaxies, find that the $z=8-10$ LF may be more like the $z=3$ LF by Steidel et al. (1999). In this case, the chances of finding a $J$-dropout LBG in the NICMOS Parallel Imaging Survey are better, with about $1-10 \mathrm{deg}^{-2}$ in both the Schechter and broken power-law forms.

The LF constraint that we estimated from the absence of $J$ dropout galaxies with $J_{110}-H_{160} \geq 2.5$ in the Bouwens et al. (2005) survey suggests they are too few and too faint for any to be found in the NICMOS Parallel Imaging Survey. However, Bouwens et al. survey only a small volume for rare objects, and so the cosmic variance will be large. Alternatively, the results of Yan et al. (2006) and Eyles et al. (2007) show that to produce the observed mass density at $z=6$, the progenitors at $z>7$ must have formed stars more rapidly in the past. The magnitude of this brightening is uncertain, but Eyles et al. (2007) suggest it is a factor of a few. We show this inferred LF, as 2 times brighter than the $z=6$ measurement by Bouwens et al. (2006) with no density evolution. This predicted LF is similar to the measurement by Richard et al. (2006) again, suggesting that we expect, at best, a $40 \%$ chance of finding a $J$-dropout LBG. If the brightening in the LF is instead a factor of 4 larger than at $z=6$, then we can expect one or two $J$ - dropout LBGs. In addition, we find that more than a factor of 10 increase in $L^{*}$ (with no density evolution) is unlikely, since we would then expect more than 16 galaxies with $J_{110}-$ $H_{160} \geq 2.5$ in this survey.

\subsection{Star Formation and Reionization at $z \geq 8$}

$J$-dropout galaxies may play an important role in the reionization of the universe. The critical star formation rate density $\left(\rho_{\mathrm{SFR}}\right)$ required to ionize the universe is $\rho_{\mathrm{SFR}}(z=9.5)=$ $0.09 M_{\odot} \mathrm{yr}^{-1} \mathrm{Mpc}^{-3}$ (Madau et al. 1999), if all ionizing photons escape the galaxies, and the clumping factor of ionized hydrogen is 30. These parameters are uncertain, but this is much larger than the upper limit posed by Bouwens et al. (2005) for galaxies at $z \sim 10$. Alternatively, for the LF, which predicts a few $J$-dropout LBGs in this survey, where $L_{z=9.5}^{*}=4 L_{z=6}^{*}$, and $\phi_{z=9.5}^{*}=\phi_{z=6}^{*}$, we calculate $\rho_{\mathrm{SFR}}(z=9.5) \sim 0.1 \quad M_{\odot} \mathrm{yr}^{-1}$ $\mathrm{Mpc}^{-3}$ in both the Schechter and broken power-law forms. In this case, $J$-dropout LBGs may be capable of reionizing the universe, although the uncertainties in the escape fraction of ionizing photons and the clumping factor of ionized hydrogen must be addressed.

To conclude, it is not implausible that $\rho_{\mathrm{SFR}}$ is very large during the first few hundred million years of galaxy formation if stars were formed in rapid bursts. In fact, confirmation of any $J$ dropout galaxies will suggest a large $\rho_{\mathrm{SFR}}$ for most reasonable $H^{*}(z=9.5)$. Follow-up observations of the galaxies presented here are one way to constrain star formation at $z \geq 8$.

This research was supported by NASA through Hubble Space Telescope Guest Observer grants 9865 and 10226. We are grateful to R. Thompson for helpful comments that improved this manuscript.

\section{REFERENCES}

Bertin, E., \& Arnouts, S. 1996, A\&AS, 117, 393

Bouwens, R. J., Illingworth, G. D., Blakeslee, J. P., \& Franx, M. 2006, ApJ, 653,53

Bouwens, R. J., Illingworth, G. D., Thompson, R. I., \& Franx, M. 2005, ApJ, 624, L5

Bruzual, G., \& Charlot, S. 2003, MNRAS, 344, 1000

Cardelli, J. A., Clayton, H. C., \& Mathis, J. S. 1989, ApJ, 345, 245

Daddi, E., et al. 2005, ApJ, 631, L13

Dickinson, M. 1999, in AIP Conf. Proc. 470, After the Dark Ages: When Galaxies Were Young, ed. S. S. Holt \& E. P. Smith (New York: AIP), 122 Dickinson, M., et al. 2000, ApJ, 531, 624

Eyles, L. P., Bunker, A. J., Ellis, R. S., Lacy, M., Stanway, E. R., Stark, D. P., \& Chiu, K. 2006, MNRAS, 374, 910

Fruchter, A., \& Hook, R. N. 1997, Proc. SPIE, 3164, 120

Goldader, J. D., Meurer, G., Heckman, T. M., Seibert, M., Sanders, D. B., Calzetti, D., \& Steidel, C. C. 2002, ApJ, 568, 651

Henry, A. L., Colbert, J. W., Malkan, M. A., McCarthy, P. J., Teplitz, H. I., \& Yan, L. 2005, BAAS, 37, 1194

Kashikawa, N., et al. 2006, ApJ, 637, 631

Kron, R. G. 1980, ApJS, 43, 305

Madau, P., Haardt, F., \& Rees, M. J. 1999, ApJ, 514, 648

McCarthy, P. J., et al. 1999, ApJ, 520, 548

Richard, J., Pello, R., Schaerer, D., Le Borgne, J.-F., \& Kneib, J.-P. 2006, A\&A, 456, 861
Spergel, D. N., et al. 2006, ApJ, in press (astro-ph/0603449)

Steidel, C. C., Adelberger, K. L., Giavalisco, M., Dickinson, M., \& Pettini, M. 1999, ApJ, 519, 1

Surace, J. A., Sanders, D. B., \& Evans, A. S. 2000, ApJ, 529, 170

Teplitz, H. I., Gardner, J. P., Malumuth, E. M., \& Heap, S. R. 1998, ApJ, 507, L17

Thompson, R. I. 2003, ApJ, 596, 748

Thompson, R. I., Eisenstein, D., Fan, X., Dickinson, M., Illingworth, G., \& Kennicut, R. C., Jr. 2006, ApJ, 647, 787

Thompson, R. I., Rieke, M., Schneider, G., Hines, D. C., \& Corbin, M. R. 1998, ApJ, 492, L95

Thompson, R. I., Storrie-Lombardi, L. J., Weymann, R. J., Reike, M. J., Schneider, G., Stobie, E., \& Lytle, D. 1999, AJ, 117, 17

Thompson, R. I., et al. 2005, AJ, 130, 1

Yan, H., Dickinson, M., Giavalisco, M., Stern, D., Eisenhardt, P. R. M., \& Ferguson, H. C. 2006, ApJ, 651, 24

Yan, L., McCarthy, P. J., Freudling, W., Teplitz, H. I., Malumuth, E. M., Weymann, R. J., \& Malkan, M. A. 1999, ApJ, 519, L47

Yan, L., McCarthy, P. J., Storrie-Lombardi, L. J., \& Weymann, R. J. 1998, ApJ, 503, L19

Yan, L., McCarthy, P. J., Weymann, R. J., Malkan, M. A., Teplitz, H. I., Storrie-Lombardi, L. J., Smith, M., \& Dressler, A. 2000, AJ, 120, 575

Yan, L., et al. 2005, ApJ, 628, 604

Yoshida, M., et al. 2006, ApJ, 653, 988 